Specificity was $82.8 \%$ for mild vs. moderate/severe, but $36.7 \%$ for mild/moderate vs. severe.

Conclusions The Garris algorithm, developed for use in administrative datasets, has acceptable performance for classifying SLE severity when compared to the gold standard of highest SLEDAI-2K assessment in 1 year in a Lupus Center. It may be used to classify patients in administrative datasets according to their SLE severity over 1 year.

Funding Source(s): Dr. Costenbaders research is supported by NIAMS R01 AR057327 and K24 AR066109.

\section{EPIDEMIOLOGY OF ANTIPHOSPHOLIPID SYNDROME: A POPULATION-BASED STUDY}

Ali Duarte-Garcia*, Michael Pham, Cynthia Crowson, Kevin Moder, Rajiv Pruthi, Kenneth Warrington, Eric Matteson. Mayo Clinic

10.1136/lupus-2019-Ism.189

Background The epidemiology of definite antiphospholipid syndrome (APS) in the general population has not been described. A recent meta-analysis concluded that it was difficult to determine the frequency of a clinically significant antiphospholipid (aPL) profile in patients with aPL-related clinical outcomes due to the lack of robust data; only $4 \%$ of the studies had the current cutoff values for anticardiolipin antibodies $(\mathrm{aCL})$ and less than one fifth of them had confirmation after 6-12 weeks. This study aimed to characterize the epidemiology of definite APS based on the 2006 updated international consensus (Sydney) classification criteria.

Methods An inception cohort of patients with incident APS in 2000-2015 in a geographically well-defined population were identified based on comprehensive individual medical record review. All cases met the definite 2006 Sydney consensus APS criteria, including the laboratory and clinical criteria as well as laboratory confirmation after 12 weeks. Lupus anticoagulant, $\operatorname{IgM}$ and $\operatorname{IgG}$ aCL and anti-2 glycoprotein-1 antibodies were tested in a centralized lab (cutoff $>40 \mathrm{GPL} / \mathrm{MPL}$ ). Incidence rates were age and sex adjusted to the US white 2010 population. Prevalence estimates were obtained from the incidence rates assuming no increased mortality associated with APL and assuming migration in/out of the area was independent of disease status.

Results In 2000-2015, 33 cases of incident APS by the Sydney criteria were identified (mean age 54.2 years, 55\% female; $97 \%$ Caucasian). The annual incidence of APS was $2.1(95 \%$ confidence interval [CI]: 1.4-2.8) per 100000 population aged 18 years. Incidence rates were similar in both sexes. The estimated prevalence of APS was 50 per 100000 (95\% CI: 42-58) and was similar in both sexes. Six (18\%) patients had a concurrent diagnosis of systemic lupus erythematosus (SLE). The most frequent clinical manifestation was deep venous thrombosis. The overall mortality of patients with APS was not significantly different from the general population (standardized mortality ratio: 1.61 ; 95\% CI: 0.74-3.05).

Conclusions Results from this first ever population based study revealed that definite APS occurred in about 2 persons per 100000 per year. The estimated prevalence is 50 per 100000 . Overall mortality was not different from the general population. The incidence and prevalence of APS in the same population was at least as common as SLE.

Funding Source(s): None

\section{OPPOSITE PROFILES OF COMPLEMENT IN ANTIPHOSPHOLIPID SYNDROME (APS) AND SYSTEMIC LUPUS ERYTHEMATOSUS (SLE) AMONG PATIENTS WITH ANTIPHOSPHOLIPID ANTIBODIES}

${ }^{1}$ Chack-Yung Yu* ${ }^{*}$ Stephanie Savelli, ${ }^{2}$ Robert Roubey, ${ }^{3}$ Kathryn Kitzmiller, ${ }^{1}$ Danlei Zhou, ${ }^{4}$ Haikady Nagaraja, ${ }^{1}$ Evan Mulvihill, ${ }^{1}$ Fatima Barbar-Smiley, ${ }^{5}$ Stacy P Ardoin, ${ }^{6}$ Yee-Ling Wu. ${ }^{1}$ Nationwide Children's Hospital and The Ohio State University; ${ }^{2}$ Division of Rheumatology, Allergy and Immunology, The University of North Carolina at Chapel Hill, Chapel Hill, NC; ${ }^{3}$ Grand Canyon University (and The Ohio State University); ${ }^{4}$ The Ohio State University; ${ }^{5}$ Nationwide Children's Hospital; ${ }^{6}$ Nationwide Children's Hospital and The Ohio State University and the Loyola University Chicago

10.1136/lupus-2019-Ism. 190

Background Antiphospholoipid syndrome (APS) is the association of antiphospholipid antibodies (aPL) with thromboses and/or recurrent pregnancy loss (RPL). Among patients with SLE, one-third have aPL and 10\%-15\% have a manifestation of secondary APS. Although mouse models suggested complement drives the pathogenesis of APS, we have little knowledge on how complement proteins and genes contribute to the pathology of human APS, and the concurrence of SLE and APS.

Methods We performed a cross-sectional study on complement proteins and genes in 525 patients with aPL. Among them, 237 experienced thromboses and 293 had SLE; 111 had both SLE and thromboses, and 106 had neither SLE nor thrombosis. Complement protein levels were determined by radial immunodiffusion for $\mathrm{C} 4, \mathrm{C} 3$ and factor $\mathrm{H}$; and by functional ELISA for mannan binding lectin (MBL). Total C4, C4A and C4B gene copy numbers (GCN) were measured by TaqManbased realtime PCR.

Results Two to six copies of C4 genes are frequently present in a diploid genome, and each copy may code for an acidic $\mathrm{C} 4 \mathrm{~A}$ or a basic $\mathrm{C} 4 \mathrm{~B}$ protein. We observed significantly (a) higher protein levels of total $\mathrm{C} 4, \mathrm{C} 4 \mathrm{~A}, \mathrm{C} 4 \mathrm{~B}, \mathrm{C} 3$ and anticardiolipin (ACLA) IgG, (b) increased frequencies of lupus anticoagulant and males, and (c) depressed levels of complement factor $\mathrm{H}, \mathrm{MBL}$ and ACLA-IgM among patients with thrombosis than those without thrombosis $(n=288)$. We also observed significantly lower GCNs of total $\mathrm{C} 4$ and C4A among aPLpositive patients with both SLE and thrombosis than others. By contrast, aPL-positive subjects with SLE had significantly reduced protein levels of $\mathrm{C} 3$, total $\mathrm{C} 4, \mathrm{C} 4 \mathrm{~A}, \mathrm{C} 4 \mathrm{~B}$ and ACLAIgG, and higher frequency of females than those without SLE. Patients with thrombosis but without SLE $(n=126)$, and patients with SLE but without thrombosis $(n=182)$ had the greatest differences in mean protein levels of $\mathrm{C} 3(\mathrm{p}=2.6 \times 10$ $6)$, C4 $(p=2.2 \times 10-9)$ and ACLA-IgG $(p=1.2 \times 10-5)$. RPL occurred in $23.7 \%$ of female patients and thrombotic SLE patients had the highest frequency of RPL $(41.0 \%$; $\mathrm{p}=3.8 \times 10-10)$. Compared with non-RPL females, RPL had significantly higher frequency of thrombosis and elevated $\mathrm{C} 4$ protein levels. Female patients with homozygous C4A deficiency all experienced RPL $(p=0.0001)$ but the opposite was true for patients with homozygous C4B deficiency $(p=0.017)$.

Conclusions There are substantial differences for complement protein concentrations and genetic diversities among aPL-positive patients with thromboses, recurrent pregnancy loss and SLE. These results are relvant for diagnosis and management of APS and SLE.

Funding Source(s): This work was supported by NIH grants 1 R01 AR050078, 1 R21070905, 1 R01 AR073311 (CYY), and RR00046 (UNC). 